Bull. Korean Math. Soc. 49 (2012), No. 1, pp. 1-10

http://dx.doi.org/10.4134/BKMS.2012.49.1.001

\title{
SOLVABILITY FOR A CLASS OF THE SYSTEMS OF THE NONLINEAR ELLIPTIC EQUATIONS
}

\author{
Tacksun Jung and Q-Heung Choi
}

\begin{abstract}
Let $\Omega$ be a bounded subset of $\mathbb{R}^{n}$ with smooth boundary. We investigate the solvability for a class of the system of the nonlinear elliptic equations with Dirichlet boundary condition. Using the mountain pass theorem we prove that the system has at least one nontrivial solution.
\end{abstract}

\section{Introduction}

Let $\Omega$ be a bounded subset of $\mathbb{R}^{n}$ with smooth boundary. Let $0<\lambda_{1}<$ $\lambda_{2} \leq \cdots \leq \lambda_{k} \leq \cdots$ be the eigenvalues of the eigenvalue problem for a single elliptic equation $-\Delta u=\lambda u$ with Dirichlet boundary condition and $\phi_{k}$ be the eigenfunction corresponding to the eigenvalue $\lambda_{k}, k \geq 1$. Let $F: \mathbb{R}^{n} \rightarrow \mathbb{R}$ be a $C^{2}$ function such that $F(0, \ldots, 0)=0$. In this paper we are concerned with the multiplicity of the solutions for a class of the system of the nonlinear elliptic equations with Dirichlet boundary condition

$$
\begin{aligned}
-\Delta u_{1}=F_{u_{1}}\left(u_{1}, \ldots, u_{n}\right) & \text { in } \Omega, \\
-\Delta u_{2}=F_{u_{2}}\left(u_{1}, \ldots, u_{n}\right) & \text { in } \Omega, \\
\vdots & \vdots \quad \vdots \quad \\
-\Delta u_{n} & =F_{u_{n}}\left(u_{1}, \ldots, u_{n}\right) \\
u_{i}(x) & =0 \quad \text { on } \partial \Omega,
\end{aligned}
$$

where $u_{i}(x) \in W_{0}^{1,2}(\Omega)$ and $F_{u_{i}}\left(u_{1}, \ldots, u_{n}\right)=\frac{\partial F\left(u_{1}, \ldots, u_{n}\right)}{\partial u_{i}}$. Let $U=\left(u_{1}, \ldots\right.$, $\left.u_{n}\right), F_{U}(U)=\operatorname{grad} F(U)=\left(F_{u_{1}}\left(u_{1}, \ldots, u_{n}\right), \ldots, F_{u_{n}}\left(u_{1}, \ldots, u_{n}\right)\right)$ and $|\cdot|$ denote the Euclidean norm in $\mathbb{R}^{n}$. Let $H$ be a Cartesian product of the Sobolev spaces $W_{0}^{1,2}(\Omega, \mathbb{R})$, i.e., $H=W_{0}^{1,2}(\Omega, \mathbb{R}) \times \cdots \times W_{0}^{1,2}(\Omega, \mathbb{R})$. We endow the

Received November 7, 2008; Revised July 1, 2011.

2010 Mathematics Subject Classification. 35J50, 35J55, 35J20, 35Q72.

Key words and phrases. system of nonlinear elliptic equations, mountain pass theorem, (P.S.) condition, critical point theory. 
Hilbert space $H$ with the norm

$$
\|U\|^{2}=\sum_{i=1}^{n}\left\|u_{i}\right\|^{2}
$$

where $\left\|u_{i}\right\|^{2}=\int_{\Omega}\left|\nabla u_{i}(x)\right|^{2} d x$.

We assume that $F$ satisfies the following conditions:

(F1) $\lim _{\left(u_{1}, \ldots, u_{n}\right) \rightarrow(0, \ldots, 0)} \frac{F_{u_{i}}(U)}{\|U\|}=0$,

(F2) $\lim _{\|U\| \rightarrow \infty} \frac{F_{u_{i}}(U)}{\|U\|}=\infty, i=1, \ldots, n$,

(F3) $U \cdot F_{U}(U) \geq \mu F(U) \forall u$,

(F4) $\left|F_{r_{1}}\left(r_{1}, \ldots, r_{n}\right)\right|+\cdots+\left|F_{r_{n}}\left(r_{1}, \ldots, r_{n}\right)\right| \leq \gamma\left(\left|r_{1}\right|^{\nu}+\cdots+\left|r_{n}\right|^{\nu}\right)$, $\forall r_{1}, \ldots, r_{n}$, where $\left.\gamma \geq 0, \mu \in\right] 2,2^{*}\left[, \nu \leq 2^{*}-1-\left(2^{*}-\mu\right)\left(1-\frac{2^{* \prime}}{2^{*}}\right)\right.$, $i=1, \ldots, n$.

Some papers of Lee $[13,16,17,18]$ concerning the semilinear elliptic system and some papers of the other several authors $[10,15]$ have treated the system of this kind nonlinear elliptic equations. In $[1,2,3,7]$ the authors studied the existence of solutions of the single elliptic equation. In $[4,5,6,8,9,11,12,14$, $19,20,21]$ the authors used variational methods and critical point theory for the existence and multiplicity of solutions of boundary value problems.

System (1.1) can be rewritten by

$$
\begin{aligned}
-\Delta U & =\nabla F(U) \text { in } \Omega, \\
U & =0 \text { on } \partial \Omega .
\end{aligned}
$$

In this paper we are looking for the weak solutions of the system (1.1) in $H$, that is, $U=\left(u_{1} \ldots, u_{n}\right) \in H$ such that

$$
\int_{\Omega}[-\Delta U \cdot V] d x-\int_{\Omega} F_{U}(U) \cdot V=0 \text { for all } V \in H,
$$

where $F_{U}(U)=\nabla F(U)=\left(F_{u_{1}}(U), \ldots, F_{u_{n}}(U)\right)$.

Our main result is the following:

Theorem 1.1. Assume that $F$ satisfies the conditions (F1)-(F4). Then the system (1.1) has at least one nontrivial solution.

For the proof of Theorem 1.1 we approach the variational method and use the generalization of the mountain pass theorem. In Section 2, we obtain some results on the operator $-\Delta$ on $W_{0}^{1,2}(\Omega), F$, the functional $I$ on $H$, and recall the generalization of the mountain pass theorem. In Section 3, we prove Theorem 1.1 by the mountain pass theorem.

\section{Some results on $-\Delta, F, I$ and generalized mountain pass theorem}

In this section we obtain some results on the operator $-\Delta$ on $W_{0}^{1,2}(\Omega)$, $F$, the functional $I$ on $H$, and recall the generalization of the mountain pass theorem. Since $\lambda_{i}>0$ for all $i \geq 1$, we have the following lemma. 
Lemma 2.1. Let $u \in W_{0}^{1,2}(\Omega, \mathbb{R})$ and $\|\cdot\|$ be a Sobolev norm. Then

(i) $\|u\| \geq C\|u\|_{L^{2}(\Omega)}$ for some constant $C>0$,

(ii) $\|u\|=0$ if and only if $\|u\|_{L^{2}(\Omega)}=0$,

(iii) $-\Delta u \in W_{0}^{1,2}(\Omega, \mathbb{R})$ implies $u \in W_{0}^{1,2}(\Omega, \mathbb{R})$.

Proof. (i) Let $\lambda_{j}$ be an eigenvalue of the eigenvalue problem for a single elliptic equation $-\Delta u=\lambda u$ in $\Omega$ with Dirichlet boundary condition. If $u \in W_{0}^{1,2}(\Omega, \mathbb{R})$, then $u$ can be expressed by

Thus we have that

$$
u=\sum c_{j} \phi_{j}
$$

$$
\|u\|^{2}=\int_{\Omega}|\nabla u(x)|^{2} d x=\int_{\Omega}(-\Delta u) u d x=\sum \lambda_{j} c_{j}^{2} \geq \lambda_{1} \sum c_{j}^{2}=\lambda_{1}\|u\|_{L^{2}(\Omega)} .
$$

Therefore we have that $\|u\|^{2} \geq C\|u\|_{L^{2}(\Omega)}$, where $C=\lambda_{1}$.

(ii) is trivial.

(iii) Let us set $f=-\Delta u \in W_{0}^{1,2}(\Omega, \mathbb{R})$. Then $f$ can be expressed by

$$
f=\sum h_{j} \phi_{j} \text {. }
$$

Then

$$
(-\Delta)^{-1} f=\sum \frac{1}{\lambda_{j}} h_{j} \phi_{j}
$$

Hence we have the inequality

$$
\|u\|^{2}=\left\|(-\Delta)^{-1} f\right\|^{2}=\sum \lambda_{j}^{2} \frac{1}{\lambda_{j}^{2}} h_{j}^{2}=\sum h_{j}^{2},
$$

which means that

$$
\left\|(-\Delta)^{-1} f\right\|=\|f\|_{L^{2}(\Omega)} .
$$

From Lemma 2.1, we have:

Lemma 2.2. Let $\nabla F(U) \in H=W_{0}^{1,2}(\Omega, \mathbb{R}) \times \cdots \times W_{0}^{1,2}(\Omega, \mathbb{R})$. Then all the solutions of

$$
-\Delta U=\nabla F(U)
$$

belong to $H$.

Now we return to the case of the system. We observe that by the following Proposition 2.1, the weak solutions of system (1.1) coincide with the critical points of the associated functional $I$

$$
\begin{gathered}
I \in C^{1,1}(H, \mathbb{R}), \\
I(U)=\int_{\Omega}\left[\frac{1}{2}|\nabla U|^{2}-F(x, U)\right] d x,
\end{gathered}
$$

where $U=\left(u_{1}, \ldots, u_{n}\right)$ and $|\nabla U|^{2}=\sum_{i=1}^{n}\left|\nabla u_{i}\right|^{2}, n \geq 1$. 
Proposition 2.1. Assume that the conditions (F1)-(F4) hold. Then the functional $I(u)$ is continuous, Fréchet differentiable in $H$ with Fréchet derivative

$$
\nabla I(U) V=\int_{\Omega}\left[(-\Delta U) \cdot V-F_{U}(U) \cdot V\right] d x .
$$

Moreover $D I \in C$. That is $I \in C^{1}$.

Proof. First we prove that $I(U)$ is continuous in $H$. For $U, V \in H$,

$$
\begin{aligned}
|I(U+V)-I(U)|=\mid & \frac{1}{2} \int_{\Omega}(-\Delta U-\Delta V) \cdot(U+V) d x-\int_{\Omega} F(U+V) d x \\
& -\frac{1}{2} \int_{\Omega}(-\Delta U) \cdot U d x+\int_{\Omega} F(U) d x \mid \\
= & \mid \frac{1}{2} \int_{\Omega}[(-\Delta U \cdot V-\Delta V \cdot U-\Delta V \cdot V) d x \\
& -\int_{\Omega}(F(U+V)-F(U)) d x \mid .
\end{aligned}
$$

Let $u_{l}=\sum h_{j}^{l} \phi_{j}, v_{l}=\sum k_{j}^{l} \phi_{j}(l=1, \ldots, n)$. Then we have

$$
\begin{aligned}
& \left|\int_{\Omega}\left(-\Delta u_{l}\right) \cdot v_{l} d x\right|=\left|\sum \lambda_{j} h_{j}^{l} k_{j}^{l}\right| \leq\left\|u_{l}\right\| \cdot\left\|v_{l}\right\|, \\
& \left|\int_{\Omega}\left(-\Delta v_{l}\right) \cdot u_{l} d x\right|=\left|\sum \lambda_{j} k_{j}^{l} h_{j}^{l}\right| \leq\left\|u_{l}\right\| \cdot\left\|v_{l}\right\|, \\
& \left|\int_{\Omega}\left(-\Delta v_{l}\right) \cdot v_{l} d x\right|=\left|\sum \lambda_{j} k_{j}^{l} k_{j}^{l}\right| \leq\left\|v_{l}\right\|^{2},
\end{aligned}
$$

from which we have

$$
\left|\frac{1}{2} \int_{\Omega}(-\Delta U \cdot V-\Delta V \cdot U-\Delta V \cdot V) d x\right| \leq C\left(\|U\| \cdot\|V\|+\|V\|^{2}\right)
$$

for some $C>0$. By the differentiability of $F$,

$$
F(U+V)-F(U)=F_{U}(U) V+o(|V|),
$$

so we have

$$
\begin{aligned}
\left|\int_{\Omega}(F(U+V)-F(U)) d x\right| & \leq\left\|F_{U}(U)\right\|_{L^{2}(\Omega)}\|V\|_{L^{2}(\Omega)}+\int_{\Omega} o(|V|) d x \\
& \leq\left\|F_{U}(U)\right\|\|V\|+o(\|V\|) .
\end{aligned}
$$

Thus we have

$$
|I(U+V)-I(U)| \leq\left(C\|U\|+\left\|F_{U}(U)\right\|\right)\|V\|+o(\|V\|)+C\|V\|^{2},
$$

so $I(U)$ is continuous at $U$.

Next we will prove that $I(U)$ is Fréchet differentiable in $H$ with Fréchet derivative $\nabla I(U)$. For $U, V \in H$,

$$
|I(U+V)-I(U)-\nabla I(U) V|
$$




$$
\begin{aligned}
=\mid & \mid \frac{1}{2} \int_{\Omega}(-\Delta U-\Delta V) \cdot(U+V) d x-\int_{\Omega} F(U+V) d x \\
& -\frac{1}{2} \int_{\Omega}(-\Delta U) \cdot U d x+\int_{\Omega} F(U) d x-\int_{\Omega}\left(-\Delta U-F_{U}(U)\right) \cdot V d x \mid \\
= & \left|\frac{1}{2} \int_{\Omega}[-\Delta V \cdot V] d x-\int_{\Omega}\left[F(U+V)-F(U)-F_{U}(U) \cdot V\right] d x\right| .
\end{aligned}
$$

By the differentiability of $F, F(U+V)-F(U)=F_{U}(U) V+o(|V|)$, so we have

$$
\left.F(U+V)-F(U)-F_{U}(U)\right) \cdot V=o(|V|) .
$$

Thus we have

$$
\left|\int_{\Omega}\left[F(U+V)-F(U)-F_{U}(U) V\right] d x\right| \leq\left|\int_{\Omega} o(|V|) d x\right|=o(\|V\|) .
$$

Thus we have

$$
|I(U+V)-I(U)-\nabla I(U) V|=O\left(\|V\|^{2}\right) .
$$

Similarly, it is easily checked that $I \in C^{1}$.

Proposition 2.2. Assume that $F$ satisfies the conditions (F1)-(F4). Then there exist $a_{0}>0, b_{0} \in \mathbb{R}$ and $\mu>2$ such that

$$
F(U) \geq a_{0}|U|^{\mu}-b_{0}, \quad \forall U .
$$

Proof. Let $U$ be such that $|U|^{2} \geq R^{2}$. Let us set $\varphi(\xi)=F(\xi U)$ for $\xi \geq 1$. Then

$$
\varphi^{\prime}(\xi)=U \cdot F_{U}(\xi U) \geq \frac{\mu}{\xi} \varphi(\xi)
$$

Multiplying by $\xi^{-\mu}$, we get

$$
\left(\xi^{-\mu} \varphi(\xi)\right)^{\prime} \geq 0,
$$

hence $\varphi(\xi) \geq \varphi(1) \xi^{\mu}$ for $\xi \geq 1$. Thus we have

$$
F(U) \geq F\left(\frac{R|U|}{\sqrt{|U|^{2}}}\right)\left(\frac{\sqrt{|U|^{2}}}{R}\right)^{\mu} \geq c_{0}\left(\frac{\sqrt{|U|^{2}}}{R}\right)^{\mu} \geq a_{0}|U|^{\mu}-b_{0}
$$

for some $a_{0}, b_{0}$, where $c_{0}=\inf \left\{\left.F(U)|| U\right|^{2}=R^{2}\right\}$.

Proposition 2.3. Assume that $F$ satisfies the conditions (F1)-(F4). Then if $\left\|U_{j}\right\| \rightarrow+\infty$ and

$$
\frac{\int_{\Omega} U_{j} \cdot F_{U}\left(U_{j}\right) d x-2 \int_{\Omega} F\left(U_{j}\right) d x}{\left\|U_{j}\right\|} \rightarrow 0,
$$

then there exist $\left(U_{h_{j}}\right)_{j}$ and $W \in H$ such that

$$
\frac{\operatorname{grad}\left(\int_{\Omega} F\left(U_{h_{j}}\right) d x\right)}{\left\|U_{h_{j}}\right\|} \rightarrow W \text { and } \frac{U_{h_{j}}}{\left\|U_{h_{j}}\right\|} \rightarrow(0, \ldots, 0) .
$$


Proof. By (F3) and Proposition 2.2, for $U \in H$,

$$
\begin{aligned}
\int_{\Omega}\left[u \cdot F_{U}(U)\right] d x-2 \int_{\Omega} F(U) d x & \geq(\mu-2) \int_{\Omega} F(U) d x \\
& \geq(\mu-2)\left(a_{0}\|U\|_{L^{\mu}}^{\mu}-b_{1}\right) .
\end{aligned}
$$

By (F4),

$$
\left\|\operatorname{grad}\left(\int_{\Omega} F(U) d x\right)\right\| \leq C^{\prime}\left\||U|^{\nu}\right\|_{L^{2^{* \prime}}}
$$

for suitable constant $C^{\prime}$. To get the conclusion it suffices to estimate $\left\|\frac{|U|^{\nu}}{\|U\|}\right\|_{L^{2^{*}}}$ in terms of $\frac{\|U\|_{L \mu}^{\mu}}{\|U\|}$. If $\mu \geq 2^{* \prime} \nu$, then this is a consequence of Hölder inequality. Next we consider the case $\mu<2^{* \prime} \nu$. By the assumptions $\mu$ and $\nu$,

$$
\nu \leq 2^{*}-1-\left(2^{*}-\mu\right)\left(1-\frac{2^{* \prime}}{2^{*}}\right) .
$$

By the standard interpolation arguments, it follows that

$$
\left\|\frac{|U|^{\nu}}{\|U\|}\right\|_{L^{2^{*}}} \leq C\left(\frac{\|U\|_{L^{\mu}}^{\mu}}{\|U\|}\right)^{\frac{\nu \alpha}{\mu}}\|U\|^{\beta},
$$

where $\alpha$ is such that $\frac{\alpha}{\mu}+\frac{1-\alpha}{2^{*}}=\frac{1}{2^{* \prime} \nu}(\alpha>0)$ and $\beta=(1-\alpha) \nu-1-\frac{\nu \alpha}{\mu}$. By (2.5), $\beta \leq 0$. Thus we prove the proposition.

For finding at least one nontrivial solution we shall use the following generalization of the mountain pass theorem (cf. Theorem 5.3 of [22]).

Lemma 2.3 (Generalization of the Mountain Pass Theorem). Let $H$ be a real Banach space with $H=V \oplus X$, where $V$ is finite dimensional. Suppose that

(I1) $I \in C^{1}(H, \mathbb{R})$,

(I2) there are constants $\rho, \alpha>0$ and a ball $B_{\rho}$ with radius $\rho$ such that

$$
I_{\partial B_{\rho} \cap X} \geq \alpha
$$

and

(I3) there is an $e \in \partial B_{1} \cap X$ and $R>\rho$ such that if $Q \equiv\left(\bar{B}_{R} \cap V\right) \oplus\{r e \mid 0<$ $r<R\}$, then

$$
\left.I\right|_{u \in \partial Q} \leq 0
$$

(I4) I satisfies the (P.S.) condition.

Then I possesses a critical value $c \geq \alpha$ which can be characterized as

$$
c=\inf _{\gamma \in \Gamma} \max _{u \in Q} I(\gamma(u)),
$$

where

$$
\Gamma=\{\gamma \in C(\bar{Q}, H) \mid \gamma=i d \text { on } \partial Q\}
$$




\section{Proof of Theorem 1.1}

From now on we shall show that $I$ satisfies the conditions (I1)-(I4) under the assumptions (F1)-(F4). Assume that the (F1)-(F4) hold.

We have the following inequalities:

Lemma 3.1. Assume that $F$ satisfies the conditions (F1)-(F4). Let $V_{i}$ be the finite dimensional subspace of $W_{0}^{1,2}(\Omega)$ spanned by eigenfunctions corresponding to the eigenvalues $\lambda<\lambda_{k_{i}}$, for some $k_{i} \geq 1(i=1, \ldots, n)$. Let us set

$$
V=V_{1} \times \cdots \times V_{n} .
$$

Then $V$ is a subspace of $H$ and $H=V \oplus V^{\perp}$. Let us set $X=V^{\perp}$. Then

(i) there exist $\rho>0$ and a small ball $B_{\rho}$ with radius $\rho$ such that

$$
\inf _{U \in \partial B_{\rho} \cap X} I(U)>0, \quad \inf _{U \in B_{\rho} \cap X} I(U)>-\infty
$$

and

(ii) there exist $e \in \partial B_{1} \cap X$ and $Q \equiv\left(\bar{B}_{R} \cap V\right) \oplus\{r e \mid 0<r<R\}$ such that

$$
\sup _{U \in \partial Q} I(U)<0 \text {. }
$$

Proof. First we will prove that there exist $\rho>0$ and a ball $B_{\rho}$ with radius $\rho$ such that $B_{\text {rho }} \cap X \neq \emptyset$ and $\inf _{U \in \partial B_{\rho} \cap X} I(U)>0$. Let $U \in X$. Then we have that

$$
I(U)=\frac{1}{2}\|U\|^{2}-\int_{\Omega} F(U) d x
$$

By (F3) and (F4), $F(U) \leq a|U|^{\beta}, a>0$ and $\beta>2$. So we have

$$
I(U) \geq \frac{1}{2}\|U\|^{2}-a\|U\|_{L^{2}(\Omega)}^{\beta} .
$$

Since $\beta>2$, there exist a small number $\rho>0$ and a small ball $B_{\rho}$ with radius $\rho$ such that if $U \in \partial B_{\rho} \cap X$, then $\inf _{U \in \partial B_{\rho} \cap X} I(U)>0$ and $\inf _{U \in B_{\rho} \cap X} I(U)>$ $-\infty$. Next, we will prove that there exist $e \in \partial B_{1} \cap X$ and $Q=\left(\bar{B}_{R} \cap V\right) \oplus$ $\{r e \mid 0<r<R\}$ such that $\sup _{U \in \partial Q} I(U)<0$. Let us choose an element $e \in X$ with $\|e\|=1$ and $U \in V \oplus\{r e \mid r>0\}$. Let $P_{Y}$ be a projection from $H$ onto a subspace $Y$ of $H$. Then we have

$$
I(U)=\frac{1}{2} r^{2}+\frac{1}{2}\left\|P_{V} U\right\|^{2}-\int_{\Omega} F(U) d x .
$$

By Proposition 2.2, there exist $a_{0}>0, b_{0} \in \mathbb{R}$ and $\mu>2$ such that $F(U) \geq$ $a_{0}|U|^{\mu}-b_{0}, \forall u$. Thus we have

$$
I(U) \leq \frac{1}{2} r^{2}+\frac{1}{2}\left\|P_{V} U\right\|^{2}-a_{0}\|U\|_{L^{2}(\Omega)}^{\mu}+b_{0}
$$

Since $\mu>2$, there exists $R>\rho$ such that if $U \in \partial\left(\left(\bar{B}_{R} \cap V\right) \oplus\{r e \mid 0<r<R\}\right)$, then $I(U)<\frac{1}{2} R^{2}+\frac{1}{2} R^{2}-a_{0}\|U\|_{L^{2}(\Omega)}^{\mu}+b_{0}<0$. Thus we have $\sup _{U \in \partial Q} I(U)<0$, where $Q=\left(\bar{B}_{R} \cap V\right) \oplus\{r e \mid 0<r<R\}$. 
Lemma 3.2. Assume that F satisfies the conditions (F1)-(F4) hold. Then I satisfies the (P.S.) condition.

Proof. Let $c \in \mathbb{R}, j \rightarrow+\infty$ and $\left(U_{j}\right)_{j}$ be a sequence such that

$$
U_{j}=\left(u_{1}^{j}, \ldots, u_{n}^{j}\right) \in H, \forall j, I\left(U_{j}\right) \rightarrow c, \nabla I\left(U_{j}\right) \rightarrow 0 .
$$

We claim that $\left(U_{j}\right)_{j}$ is bounded. By contradiction we suppose that $\left\|U_{j}\right\| \rightarrow+\infty$ and set $\hat{U}_{j}=\frac{U_{j}}{\left\|U_{j}\right\|}$. Then

$$
\left\langle\nabla I\left(U_{U}\right), \hat{U}_{j}\right\rangle=2 \frac{I\left(U_{j}\right)}{\left\|U_{j}\right\|}-\frac{\int_{\Omega} F_{U}\left(U_{j}\right) \cdot U_{j} d x-2 \int_{\Omega} F\left(U_{j}\right) d x}{\left\|U_{j}\right\|} \longrightarrow 0 .
$$

Hence

$$
\frac{\int_{\Omega} F_{U}\left(U_{j}\right) \cdot U_{j} d x-2 \int_{\Omega} F\left(U_{j}\right) d x}{\left\|U_{j}\right\|} \longrightarrow 0 .
$$

By Proposition 2.3,

$$
\frac{\operatorname{grad} \int_{\Omega} F\left(U_{j}\right) d x}{\left\|U_{j}\right\|} \quad \text { converges }
$$

and $\hat{u_{n}} \rightarrow 0$. We get

$$
\frac{\nabla I\left(U_{j}\right)}{\left\|U_{j}\right\|}=-\Delta \hat{U}_{j}-\frac{\operatorname{grad}\left(\int_{\Omega} F\left(U_{j}\right) d x\right)}{\left\|U_{j}\right\|} \longrightarrow 0,
$$

so $-\Delta \hat{U}_{j}$ converges. Since $\left(\hat{U}_{j}\right)_{j}$ is bounded and the inverse operator of $-\Delta$ is a compact mapping, up to subsequence, $\left(\hat{U}_{j}\right)_{j}$ has a limit. Since $\hat{U}_{j} \rightarrow(0, \ldots, 0)$, we get $\hat{U}_{j} \rightarrow(0, \ldots, 0)$, which is a contradiction to the fact that $\left\|\hat{U}_{j}\right\|=1$. Thus $\left(U_{j}\right)_{j}$ is bounded. We can now suppose that $U_{j} \rightarrow U$ for some $U \in H$. Since the mapping $U \mapsto \operatorname{grad}\left(\int_{\Omega} F(U) d x\right)$ is a compact mapping, $\operatorname{grad}\left(\int_{\Omega} F\left(U_{j}\right) d x\right) \longrightarrow$ $\operatorname{grad}\left(\int_{\Omega} F(U) d x\right)$. Thus $-\Delta U_{j}$ converges. Since the inverse operator of $-\Delta$ is a compact operator and $\left(U_{j}\right)_{j}$ is bounded, we deduce that, up to a subsequence, $\left(U_{j}\right)_{j}$ converges to some $U$ strongly with $\nabla I(U)=\lim \nabla I\left(U_{j}\right)=0$. Thus we prove the lemma.

Proof of Theorem 1.1. Let $V_{i}$ be the finite dimensional subspace of $W_{0}^{1,2}(\Omega)$ spanned by eigenfunctions corresponding to the eigenvalues $\lambda<\lambda_{k_{i}}$, for some $k_{i} \geq 1(i=1, \ldots, n)$. Let us set

$$
V=V_{1} \times \cdots \times V_{n}
$$

Then $V$ is a subspace of $H$ and $H=V \oplus V^{\perp}$. Let us set $X=V^{\perp}$. By Proposition 2.1, $I$ is $C^{1}(H, \mathbb{R})$, so the condition (I1) of Lemma 2.3 is satisfied. By Lemma 3.1, the conditions (I2) and (I3) of Lemma 2.3 are satisfied. By Lemma $3.2, I(U)$ satisfies the (P.S.) condition, so the condition (I4) of Lemma 2.3 is satisfied. Thus by Lemma 2.3 , there exists at least one nontrivial critical point for $I$ whose critical value is

$$
I(U)=\inf _{\gamma \in \Gamma} \max _{U \in Q} I(\gamma(U)),
$$


where $\Gamma=\{\gamma \in C(\bar{Q}, H) \mid \gamma=i d$ on $\partial Q\}$. Thus we prove the theorem.

Acknowledgements. The second author was supported by Inha University Research Grant. The authors appreciate very much for the referee's kind corrections.

\section{References}

[1] A. Ambrosetti and P. Rabinowitz, Dual variational methods in critical point theory and applications, J. Funct. Anal. 14 (1973), 349-381.

[2] A. Castro and J. Cossio, Multiple solutions for a nonlinear Dirichlet problem, SIAM J. Math. Anal. 25 (1994), no. 6, 1554-1561.

[3] A. Castro and A. C. Lazer, Critical point theory and the number of solutions of a nonlinear Dirichlet problem, Ann. Mat. Pura Appl. (4) 120 (1979), 113-137.

[4] K. C. Chang, Solutions of asymptotically linear operator equations via Morse theory, Comm. Pure Appl. Math. 34 (1981), no. 5, 693-712.

[5] - Infinite Dimensional Morse Theory and Multiple Solution Problems, Birkhäuser, 1993.

[6] Q. H. Choi and T. Jung, Multiple periodic solutions of a semilinear wave equation at double external resonances, Commun. Appl. Anal. 3 (1999), no. 1, 73-84.

[7] E. N. Dancer, On the Dirichlet problem for weakly non-linear elliptic partial differential equations, Proc. Roy. Soc. Edinburgh Sect. A 76 (1976/77), no. 4, 283-300.

[8] M. Degiovanni, Homotopical properties of a class of nonsmooth functions, Ann. Mat. Pura Appl. (4) 156 (1990), 37-71.

[9] M. Degiovanni, A. Marino, and M. Tosques, Evolution equations with lack of convexity, Nonlinear Anal. 9 (1985), no. 12, 1401-1443.

[10] D. R. Dunninger and H. Wang, Multiplicity of positive radial solutions for an elliptic system on an annulus, Nonlinear Anal. 42 (2000), no. 5, Ser. A: Theory Methods, 803-811.

[11] G. Fournier, D. Lupo, M. Ramos, and M. Willem, Limit relative category and critical point theory, Dynam. Report 3 (1993), 1-23.

[12] A. Groli, A. Marino, and C. Saccon, Variational theorems of mixed type and asymptotically linear variational inequalities, Topol. Methods Nonlinear Anal. 12 (1998), no. 1, 109-136.

[13] K. S. Ha and Y. H. Lee, Existence of multiple positive solutions of singular boundary value problems, Nonlinear Anal. 28 (1997), no. 8, 1429-1438.

[14] H. Hofer, Variational and topological methods in partially ordered Hilbert spaces, Math. Ann. 261 (1982), no. 4, 493-514.

[15] K. Lan and R. L. Webb, Positive solutions of semilinear differential equations with singularities, J. Differential Equations 148 (1998), no. 2, 407-421.

[16] Y. H. Lee, Existence of multiple positive radial solutions for a semilinear elliptic system on an unbounded domain, Nonlinear Anal. 47 (2001), no. 6, 3649-3660.

[17] _ A multiplicity result of positive radial solutions for a multiparameter elliptic system on an exterior domain, Nonlinear Anal. 45 (2001), no. 5, Ser. A: Theory Methods, 597-611.

[18] _ Multiplicity of positive radial solutions for multiparameter semilinear elliptic systems on an annulus, J. Differential Equations 174 (2001), no. 2, 420-441.

[19] A. Marino and C. Saccon, Nabla theorems and multiple solutions for some noncooperative elliptic systems, Sezione Di Annalisi Mathematica E Probabilita, Dipartimento di Mathematica, Universita di Pisa, 2000.

[20] _ Some variational theorems of mixed type and elliptic problems with jumping nonlinearities, Ann. Scuola Norm. Sup. Pisa Cl. Sci. (4) 25 (1997), no. 3-4 631-665. 
[21] A. M. Micheletti and A. Pistoia, Multiplicity results for a fourth-order semilinear elliptic problem, Nonlinear Anal. 31 (1998), no. 7, 895-908.

[22] P. H. Rabinowitz, Minimax Methods in Critical Point Theory with Applications to Differential Equations, C.B.M.S. Reg. Conf. Ser. in Math. 6, American Mathematical Society, Providence, R1, 1986.

TACKSUN JunG

Department of Mathematics

Kunsan National University

KUNSAN 573-701, KOREA

E-mail address: tsjung@kunsan.ac.kr

Q-Heung Choi

Department of Mathematics Education

INHA UNIVERSITY

INCHEON 402-751, KoREA

E-mail address: qheung@inha.ac.kr 